\title{
Genetic Network Programming with Reinforcement Learning and Its Application to Making Mobile Robot Behavior
}

\author{
Shingo Mabu Member (Waseda University, mabu@asagi.waseda.jp) \\ Hiroyuki Hatakeyama Student Member (Waseda University, h_hata@toki.waseda.jp) \\ Moe Thu Thu Non-member (Waseda University, moethu@suou.waseda.jp) \\ Kotaro Hirasawa Member (Waseda University, hirasawa@waseda.jp) \\ Jinglu $\mathrm{Hu}$ Member (Waseda University, jinglu@waseda.jp)
}

Keywords: evolutionary computation, genetic network programming, reinforcement learning, khepera robot

A new graph-based evolutionary algorithm called "Genetic Network Programming (GNP)" has been proposed. GNP represents its solutions as graph structures which have some distinguished abilities. For example, GNP can memorize past action sequences in the network flow and make compact structures. However, conventional GNP is based on evolution, i.e., after GNP programs are carried out to some extent, they are evaluated and evolved based on their fitness values, so many trials must be executed again and again. Therefore, GNP with Reinforcement Learning (GNP-RL) which combines evolution-based GNP and RL has been proposed. In this method, because RL is done when an agent is carrying out its task, GNP can search for better solutions every judgment and processing during task execution besides the evolutional operation executed after task execution. The aim of combining evolution and RL is to take advantage of the sophisticated diversified search ability of evolution and the intensified search ability of RL with online learning. Moreover, the size of the $Q$ table could be set at small values. Therefore, the calculation time and memory consumption can be saved.

In this paper, in order to extend the ability of GNP-RL and apply to real world problems, we aim to deal with numerical information (ex. 15 degrees, 512 points, etc.), while all the algorithms of GNP already proposed deal with discrete information (ex. right, left, etc.). In the simulations, the proposed method is applied to the controller of the Khepera simulator and it learns wall-following behavior.

Wall-following behavior problem requires the robot to move along walls and also requires it to move as fast and straight as possible. The rewards and fitness values used for learning and evolution, respectively are calculated based on the above conditions.

Table 1 shows the generalization ability of GNP-RL, evolution-based GNP (standard GNP) and Neural Networks evolved by GA (NN-GA). The average fitness is calculated as follows. First, each method produces programs in a training
Table 1. Data on the fitness obtained in the testing environment

\begin{tabular}{l|ccc}
\hline & GNP-RL & S-GNP & NN-GA \\
\hline average fitness & 0.426 & 0.356 & 0.204 \\
standard deviation & 0.082 & 0.146 & 0.046 \\
\hline t-test (p-value[\%]) between & & & \\
GNP-RL and each method & - & 3.54 & $5.75 \times 10^{-10}$ \\
\hline
\end{tabular}

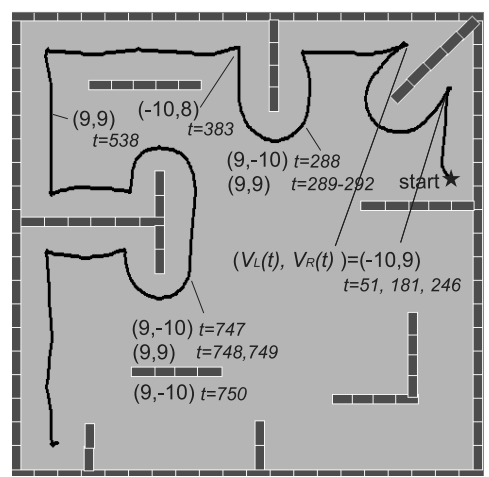

Fig. 1. Typical track of the robot controlled by GNP-RL in the testing environment

environment. Then, the average fitness is calculated in a testing environment using the best programs of each method obtained in the training environment. From Table 1, it is clarified that GNP-RL shows the best fitness and there are significant differences between GNP-RL and the other methods.

Fig. 1 shows a typical behavior of the robot controlled by GNP-RL. It also shows the speed of the wheels under several situations. From the figure, the proposed method can learn wall-following behavior well. The robot can move straight along the wall and when the wall becomes close to the robot, it can appropriately avoid colliding with the wall by adjusting the speed of the two wheels. 


\section{Genetic Network Programming with Reinforcement Learning and Its Application to Making Mobile Robot Behavior}

$\begin{array}{ll}\text { Shingo Mabu* } & \text { Member } \\ \text { Hiroyuki Hatakeyama** } & \text { Student Member } \\ \text { Moe Thu Thu } & \text { Non-member } \\ \text { Kotaro Hirasawa } & \text { Member } \\ \text { Jinglu } \mathrm{Hu}^{* *} & \text { Member }\end{array}$

A new graph-based evolutionary algorithm called “Genetic Network Programming, GNP” has been proposed. The solutions of GNP are represented as graph structures, which can improve the expression ability and performance. In addition, GNP with Reinforcement Learning (GNP-RL) has been proposed to search for solutions efficiently. GNP-RL can use current information (state and reward) and change its programs during task execution. Thus, it has an advantage over evolution-based algorithms in case much information can be obtained during task execution. The GNP we proposed in the previous research deals with discrete information, but in this paper, we extend the conventional GNP-RL which can deal with numerical information. The proposed method is applied to the controller of Khepera simulator and its performance is evaluated.

Keywords: Evolutionary Computation, Genetic Network Programming, Reinforcement Learning, Khepera robot

\section{Introduction}

A new graph-based evolutionary algorithm called "Genetic Network Programming (GNP)" has been proposed (1)-(3). In addition, an online learning algorithm of GNP using Reinforcement Learning ${ }^{(4)}$ (RL) has been proposed $^{(5)(6)}$ and combined with evolution-based GNP (GNP with RL, GNP-RL) (7) (8). In GNP-RL, since RL is carried out when an agent is carrying out its task, GNP can search for better solutions every judgment and processing during task execution besides the evolutional operation executed after task execution. Therefore, the aim of combining evolution and RL is to take advantage of the sophisticated diversified search ability of evolution and the intensified search ability of RL with online learning. Moreover, the size of the $Q$ table could be set at small values, and the calculation time and memory consumption can be saved.

In this paper, in order to extend the ability of GNP-RL and apply to real world problems, an extended algorithm of GNP-RL is proposed, which can deal with numerical input and output values efficiently. Concretely speaking, the proposed method divides an input space into two spaces using RL and determines an output value also using RL. Standard GNP can be optimized using

\footnotetext{
* Advanced Research Institute for Science and Engineering, Waseda University

Hibikino 2-2, Wakamatsu-ku, Kitakyushu, Fukuoka, 808-0135 Japan

** Graduate School of Information, Production, and Systems, Waseda University

Hibikino 2-7, Wakamatsu-ku, Kitakyushu, Fukuoka, 808-0135 Japan
}

evolution only, but GNP-RL has an advantage to do it also using online learning during task execution.

In the simulations, the proposed method is applied to the controller of Khepera simulator ${ }^{(9)}$ and the comparison between GNP-RL, standard GNP and neural network evolved by GA is carried out.

This paper is organized as follows. In the next section, the algorithm of the proposed method is described. Section 3 shows the results of the simulations. Section 4 is devoted to conclusions.

\section{Extended GNP-RL Algorithm}

Section 2.1 describes the characteristics of GNP briefly, and Section 2.2 describes the gene structure of GNP-RL used in this paper. Section 2.3 introduces new judgment and processing nodes. Section 2.4 explains the genetic operators including a new mutation operator, and Section 2.5 explains how to update Q-values.

2.1 Components Fig. 1 shows the basic structure of GNP. GNP has a number of Judgment nodes and Processing nodes. Judgment nodes have if-then type conditional branch decision functions. They return judgment results for assigned inputs and determine the next node. Processing nodes determine the actions an agent should do. While judgment nodes have conditional branches, processing nodes have no conditional branches. The graph structure of GNP has some inherent characteristics such as compact structure and implicit memory function ${ }^{(1)}$.

GNP has time delays. There are the time delays GNP spends on judgments or processing, and the ones GNP spends on node transitions. In this paper, the time de- 


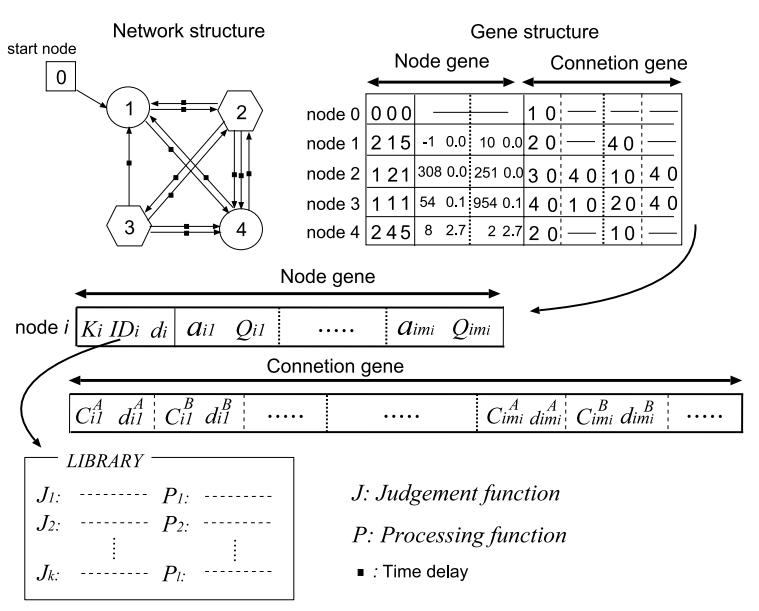

Fig. 1. Basic structure of GNP with reinforcement learning

lay of node transition is set at zero time unit, that of judgment nodes is set at one time unit, and that of processing nodes is set at five time units. In addition, one step of an agent behavior is defined in such a way that one step ends when an agent uses 10 or more time units. For example, after executing three judgments and one processing, if one processing is executed, the total used time units become 13, thus one step ends.

2.2 Gene structure of GNP The whole structure of GNP is determined by the combination of the following genes. A gene structure of node $i(0 \leq i \leq n-1$ $\dagger)$ is shown in Fig. 1. $K_{i}$ represents a node type. $K_{i}=0$ means a start node, $K_{i}=1$ means a judgment node and $K_{i}=2$ means a processing node. $I D_{i}$ shows a code number of a judgment or processing, and it is represented as a unique number shown in the LIBRARY. $d_{i}$ is a time delay spent on a judgment or processing. $a_{i p}\left(1 \leq p \leq m_{i}\right.$ $\dagger^{\dagger}$ ) is a new parameter introduced in this paper, which is described in section 2.3 in detail. Fig. 2 shows node structures with $m_{i}=2$. $Q_{i p}$ means $Q$ value which is assigned to each state and action pair. In this method, "state" means a current node, and "action" means a selection of a parameter $\left(a_{i p}\right) . C_{i p}^{A}, C_{i p}^{B}, \ldots$ show the next node number. $d_{i p}^{A}, d_{i p}^{B}, \ldots$ show time delays spent on the transition from node $i$ to the next node.

\subsection{Judgment and Processing Functions}

When a current node $i$ is a judgment node, first, one $Q$ value is selected from $Q_{i 1}, \ldots, Q_{i m_{i}}$ based on $\varepsilon$ greedy policy. That is, a maximum $Q$ value among $Q_{i 1}, \ldots, Q_{i m_{i}}$ is selected with the probability of $1-\varepsilon$, or a random one is selected with the probability of $\varepsilon$. Then the corresponding $a_{i p}$ is selected (Suppose $a_{i p}=a_{i 1}$ ). The selected $a_{i 1}$ divides input space into two spaces, i.e., when an input ${ }^{\dagger \dagger \dagger}$ is $a_{i 1}$ or more, the judgment result is $A$ and the next node number is $C_{i 1}^{A}$, or when the input is less than $a_{i p}$, the judgment result is $B$ and the

\footnotetext{
$\dagger$ Each node has a unique number from 0 to $n-1$, respectively, when the number of nodes is $n$.

$\dagger \dagger$ In this paper $m_{i}$ is set at 2 which shows the number of node parameters GNP can select at node $i$.

${ }^{\dagger \dagger}$ Input is a sensor value in this paper, and $I D_{i}$ shows the sensor number.
}

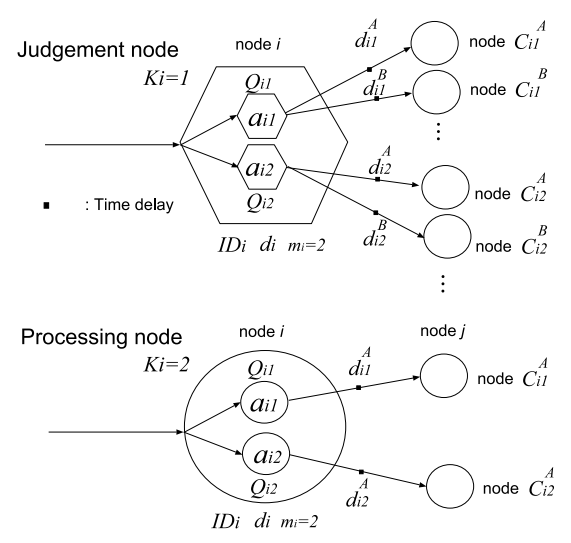

Fig. 2. Structures of nodes

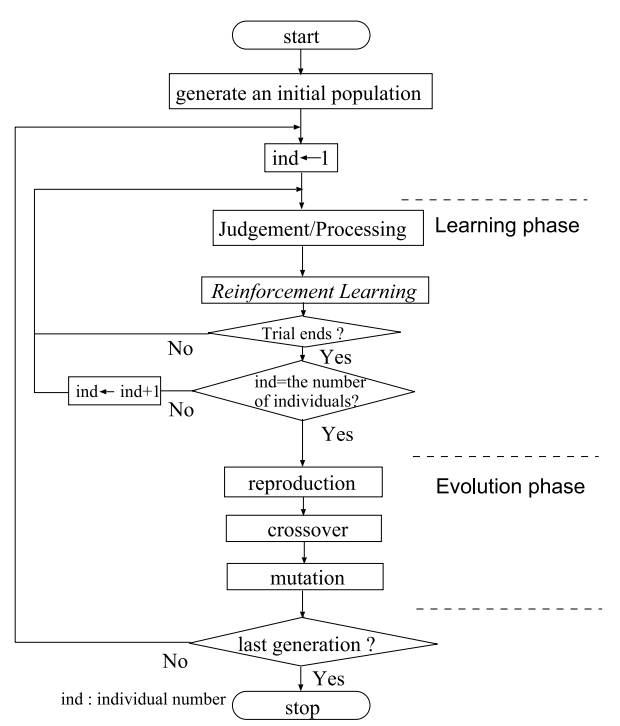

Fig. 3. Flowchart of GNP-RL

next node number is $C_{i 1}^{B}$.

When a current node is a processing node, $a_{i p}$ is selected in the same way as a judgment node (Suppose $\left.a_{i p}=a_{i 1}\right)$. In this paper, the selected $a_{i 1}$ is used to determine the speed of a robot wheel ${ }^{\dagger \dagger \dagger \dagger}$. The next node number is $C_{i 1}^{A}$.

The appropriate parameters $a_{i p}$ are determined by evolution and RL as described in section 2.4 and 2.5.

2.4 Evolution Phase Fig. 3 shows the whole flowchart of GNP-RL. In this sub-section, the genetic operators in the evolution phase are introduced. The role of evolution is to change graph structures and randomly change node parameters $a_{i p}$.

2.4.1 Crossover Crossover is executed between two parents and generates two offspring [Fig. 4].

(1) Select two parents using tournament selection.

(2) Each node $i$ is selected as a crossover node with the probability of $P_{c}$.

(3) Two parents exchange the genes of the corresponding crossover nodes (i.e., with the same node number).

${ }_{\dagger \dagger \dagger \dagger} I D_{i}=0$ : right wheel, $I D_{i}=1$ : left wheel 


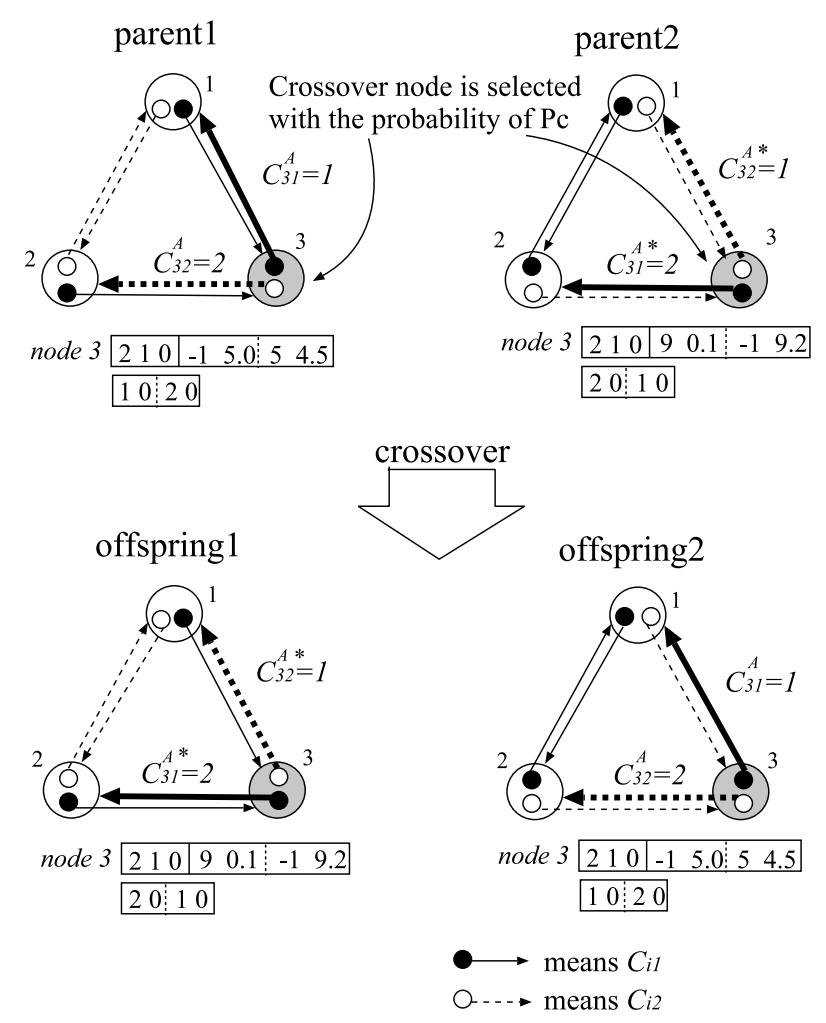

Fig. 4. Crossover

(4) Generated new individuals become the new ones in the next generation.

Fig. 4 is a simple crossover example of the graph structure with 3 processing nodes. But, when the genes of judgment nodes are exchanged, the genes with suffix $A, B, \ldots$ are exchanged simultaneously.

2.4.2 Mutation Mutation is executed in one individual and a new one is generated [Fig. 5].

(1) Select one individual using tournament selection

(2) Mutation operator

(a) connection: Each node branch is reconnected to another node with the probability of $P_{m}$.

(b) parameter: Each parameter is selected with the probability of $P_{m}$, and it is changed to another value, i.e., $a_{i p}^{\dagger}$ is changed.

(3) Generated new individual becomes the new one in the next generation.

Second operator (2-b) is a new one used in this paper. 2.5 Learning Phase In this paper, Sarsa ${ }^{(4)}$ is used to update $\mathrm{Q}$ values. Here, the procedure is explained using Fig. 6 .

(1) At time $t$, GNP refers to all $Q_{i p}$ and select one of them based on $\varepsilon$-greedy policy. We suppose that GNP selects $Q_{i p_{1}}$ and the corresponding parameter $a_{i p_{1}}$.

(2) Then GNP executes the function $I D_{i}$ using the selected $a_{i p_{1}}$, gets the reward $r_{t}$ and the next node

\footnotetext{
$\dagger a_{i p} \in[0,1023]$ (judgment node), $a_{i p} \in[-10,10]$ (processing node)
}

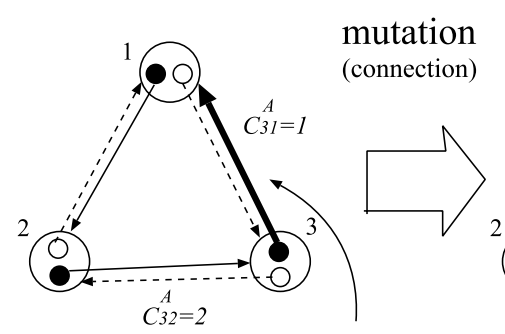

The connection is changed randomly.
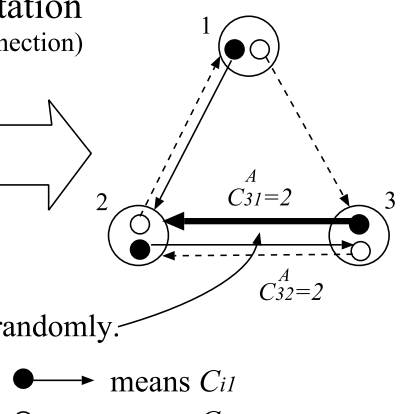

$\mathrm{O}-\ldots$ means $\mathrm{C}_{i 2}$

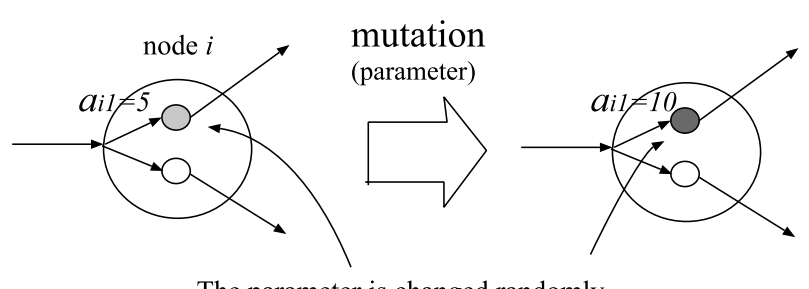

The parameter is changed randomly.

Fig. 5. Mutation

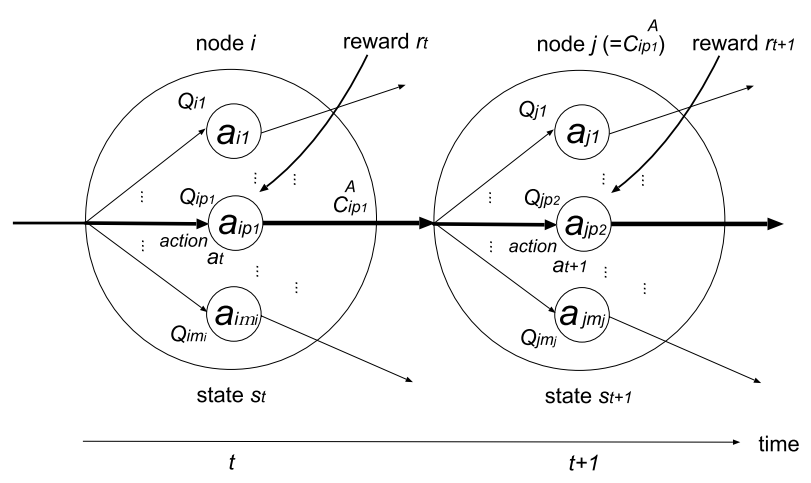

Fig. 6. An example of node transition

becomes $C_{i p_{1}}^{A}$.

(3) At time $t+1$, GNP selects one $Q_{j p}$ in the same way as step 1 . Here we suppose that $Q_{j p_{2}}$ is selected.

(4) Then the following procedure is executed.

$$
\begin{gathered}
Q_{i p_{1}} \leftarrow Q_{i p_{1}}+\alpha\left(r_{t}+\gamma Q_{j p_{2}}-Q_{i p_{1}}\right) \\
\alpha \text { : learning rate }(0<\alpha \leq 1) \\
\gamma: \text { discount } \operatorname{rate}(0 \leq \gamma \leq 1)
\end{gathered}
$$

(5) $t \leftarrow t+1, i \leftarrow j, p_{1} \leftarrow p_{2}$, then return to step 2.

In this example, node $i$ is a processing node, but if it is a judgment node, the next current node is selected among $C_{i p}^{A}, C_{i p}^{B}, \ldots$ according to the judgment result.

\section{Simulation}

In order to evaluate the ability of the proposed method, the simulations using Khepera simulator ${ }^{(9)}$ are carried out in this section.

3.1 Settings of the Robot The simulated Khepera robot [Fig. 7] includes eight infrared sensors allowing it to detect the proximity of objects in front of 


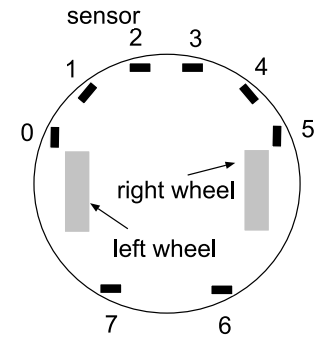

Fig. 7. Khepera robot

Table 1. Function Set

\begin{tabular}{l|l|l}
\hline \multicolumn{1}{c|}{ symbol } & \multicolumn{1}{|c|}{$I D$} & \multicolumn{1}{c}{ content } \\
\hline$J_{0}, \ldots J_{7}$ & $0, \ldots, 7$ & $\begin{array}{l}\text { judge whether the value of the sensor } \\
0, \ldots, 7 \text { is more than } a_{t} \text { or not }\end{array}$ \\
\hline$P_{0}$ & 0 & $\begin{array}{l}\text { determine the speed of the right wheel } \\
\text { determine the speed of the left wheel }\end{array}$ \\
$P_{1}$ & 1
\end{tabular}

Table 2. Simulation conditions

\begin{tabular}{l|l}
\hline the number of individuals & $\begin{array}{l}600 \text { (mutation: 359, } \\
\text { crossover: 240, elite: 1) }\end{array}$ \\
\hline the number of nodes & 60 \\
\hline parameters of evolution & $\begin{array}{l}P_{c}=0.1, P_{m}=0.01 \\
\text { tournament size }=7\end{array}$ \\
parameters of learning & $\alpha=0.9, \gamma=0.3, \varepsilon=0.1($ simulation I) \\
& $\alpha=0.1, \gamma=0.9, \varepsilon=0.01($ simulation II) \\
\hline
\end{tabular}

it, behind it, and to the right and the left side of it by reflection. Each sensor returns a value ranging between zero and 1023. Zero means that no object is perceived while 1023 means that an object is very close to the sensor (almost touching the sensor). Intermediate values may give an approximate idea of the distance between the sensor and the object. Two motors turn the right and left wheels of the robot, respectively, and they can take a speed value ranging -10 and +10 .

3.2 Settings of GNP The nodes used by GNP are shown in Table 1 . Each judgment node $\left\{J_{0}, \ldots, J_{7}\right\}$ returns $A$ or $B$ as a judgment result. For example, $J_{0}$ judges the value of the sensor $0\left(x_{0}\right)$, and if $x_{0} \geq a_{t}\left(a_{t}\right.$ is $a_{i p}$ selected at node $i$ ), the judgment result becomes $A$, and if $x_{0}<a_{t}$, it becomes $B$. Each processing node determines the speed of the left or right wheel using $a_{t}$. The parameters of evolution and learning are shown in Table 2. These values are selected appropriately through the simulations. At the end of each generation, 359 new individuals generated by mutation, 240 new individuals generated by crossover and one elite individual form the new population in the next generation. The number of judgment nodes is 40 (five each kind) and the number of processing nodes is 20 (10 each kind), therefore the total number of nodes is 60 . The initial $\mathrm{Q}$ values are set at 0 , the initial connections between nodes are determined randomly, and the initial values of $a_{i p}$ are determined randomly in the range where each parameter can take (see footnote in section 2.4.2).

3.3 Fitness and Reward (Wall-following Behavior) As described before, one step in this paper includes 10 time units (Each judgment node uses one time unit, and each processing node uses five time

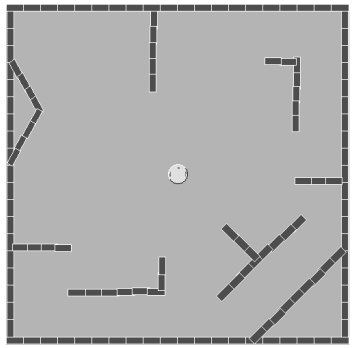

Fig. 8. Simulation environment

units.). In each step, GNP judges the values of the sensors and determines the speed of the wheels according to the node function. After that, the robot actually moves in the environment and gets reward. A trial ends when the time step reaches the predefined steps (1000), and then fitness is calculated. Fig. 8 shows the environment used in this simulation. The size of this simulated environment (comparing to the real khepera robot) is $1 \mathrm{~m} \times 1 \mathrm{~m}$ and there are the obstacles (walls) in it. In this simulation, GNP must learn the wall-following behavior, i.e., the robot must move along the wall as fast and straight as possible. Reward and fitness are calculated as follows.

$$
\begin{aligned}
\text { Reward }(t)= & \frac{v_{R}(t)+v_{L}(t)}{20} \\
& \times\left(1-\sqrt{\frac{\left|v_{R}(t)-v_{L}(t)\right|}{20}}\right) \times C, \\
\text { Fitness }= & \sum_{t=1}^{1000} \operatorname{Reward}(t), \\
\text { where, } \quad & \text { at step } t \text { respectively, } \\
v_{R}(t), v_{L}(t): & \text { the speed of right and left wheels } \\
C= & \begin{array}{ll}
1: & \text { all the sensor values are less } \\
\text { than } 1000, \text { and at least one } & \text { of them is more than } 100
\end{array}
\end{aligned}
$$

3.4 Simulation I In this simulation, the start position is fixed at the left side of the environment every trial, and the performance of the proposed method is evaluated. For the comparison, the simulation using GNP without RL (standard $\mathrm{GNP}^{\dagger}$ ) and neural networks (3-layer) evolved by GA (NN-GA) are also carried out. The reason why we use neural networks is that neural networks have a distinguished ability to deal with numerical information. The number of input nodes of NN-GA is eight (the number of sensors), that of hidden nodes is 10 , and that of output nodes is two (the speed of both wheels). The weights and thresholds of the neural networks are determined randomly first, and are updated by GA.

Fig. 9 shows the fitness curves of the best individuals at each generation averaged over 10 independent simu-

$\dagger$ Standard GNP is the same as GNP-RL with $m_{i}=1$ and without the $Q$ value update. 


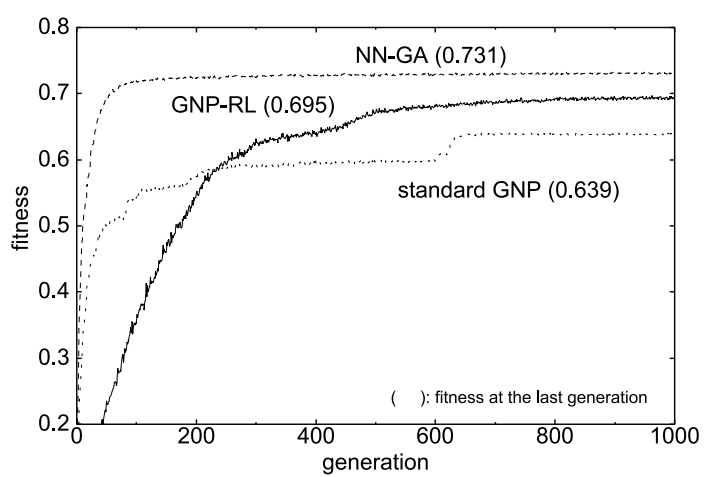

Fig. 9. Fitness curves of Simulation I

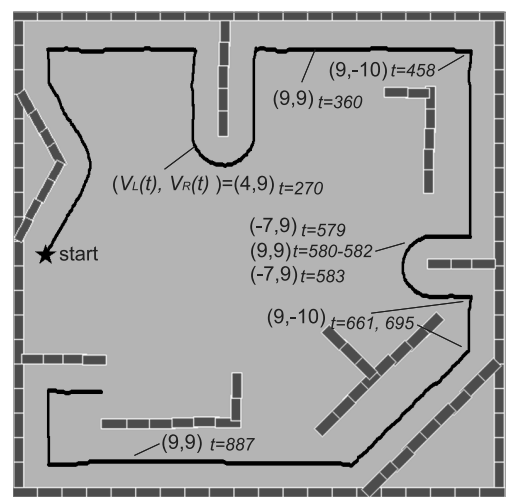

Fig. 10. Typical track of the robot controlled by GNP-RL in Simulation I

lations. From the results, NN-GA shows the best fitness in the environment with the fixed start position. Standard GNP shows better fitness than GNP-RL at the beginning of the generations because GNP-RL has plural parameters in each node and Q-values must be updated gradually, while standard GNP has only one parameter in each node. In other words, the search space of GNPRL is larger than standard GNP. However, at the end of the generation, GNP-RL shows better result than standard GNP because RL is carried out and appropriate node parameters are obtained.

Fig. 10 shows a successful behavior of the best robot controlled by GNP-RL in the last generation. It also shows the speed of the wheels under several situations. From the figure, the proposed method can learn wallfollowing behavior well. The robot can move straight along the wall and when the wall becomes close to the robot, the robot can appropriately avoid colliding with the wall by adjusting the speed of the two wheels. NNGA can also obtain good track (almost the same track as GNP-RL). Standard GNP can obtain good track in some simulations. But, in others, good results are not obtained (e.g., fitness $=0.396,0.433$ ), thus the average fitness of standard GNP becomes lower than GNP-RL.

3.5 Simulation II In this simulation, the start position is randomly changed every generation, thus if there are no walls around the robot, it must search for walls and move along them. Because the initial situation is varied every generation, GNP must do more proper judgments and processing than simulation I.

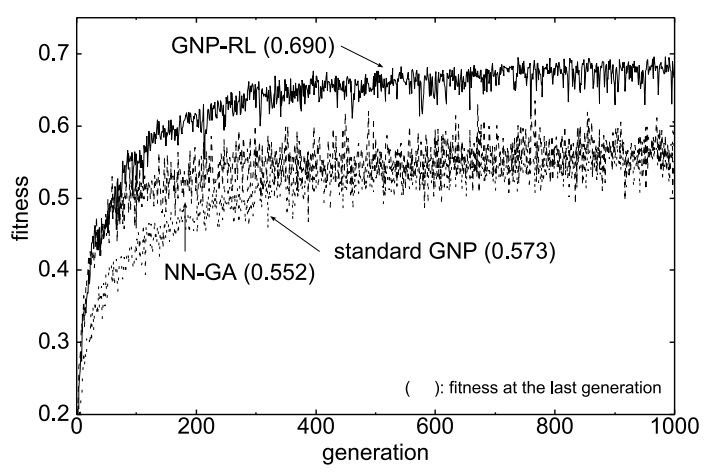

Fig. 11. Fitness Curves of Simulation II

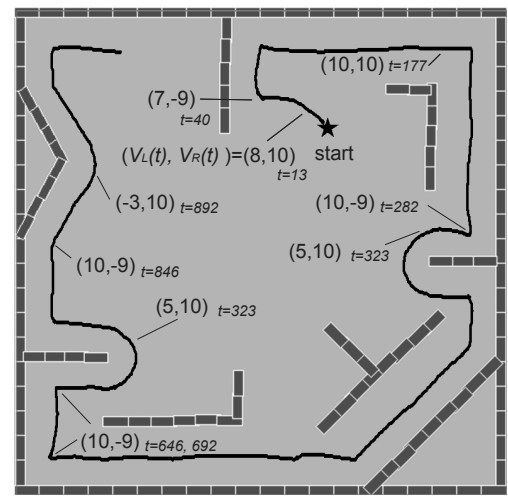

Fig. 12. Typical track of the robot controlled by GNP-RL in Simulation II

Fig. 11 shows the fitness curves averaged over 20 independent simulations, and GNP-RL shows the best fitness of the three. GNP has an ability to select necessary nodes depending on the situations, while neural networks determine their actions using all the nodes in the networks. Therefore, it can be said that GNP makes some sub-structures which can adapt to some situations. As a result, even if the start position is changed, GNP can judge its situation properly. GNP-RL shows much better fitness than standard GNP because appropriate node parameters are determined by evolution and RL. Actually, in 16 simulations out of 20 , the fitness values of GNP-RL at the last generation are more than 0.6, while those of standard GNP in 10 simulations are more than 0.6. Thus, the average fitness values of GNP-RL and standard GNP take different values. In addition, the fitness values of NN-GA in eight simulations are more than 0.6.

Fig. 12 shows a successful behavior of the robot controlled by GNP-RL. From the figure, we can see that there are no walls around the robot at first, but the robot searches for walls, and after finding walls, the robot can follow them appropriately.

3.5.1 Effect of RL Parameters In this subsection, the RL parameters are set at various values, and the effects on the fitness are examined.

Fig. 13 shows the effect of $\alpha$ on the fitness curves. Because GNP-RL must make programs considering various situations, it is necessary to update $Q$ values gradually. We can find from the figure that 0.1 is an appropriate 


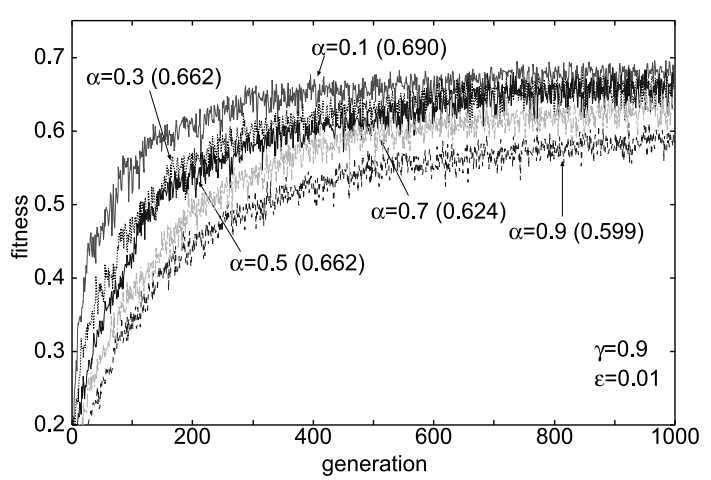

Fig. 13. effect of $\alpha$ on the fitness curves

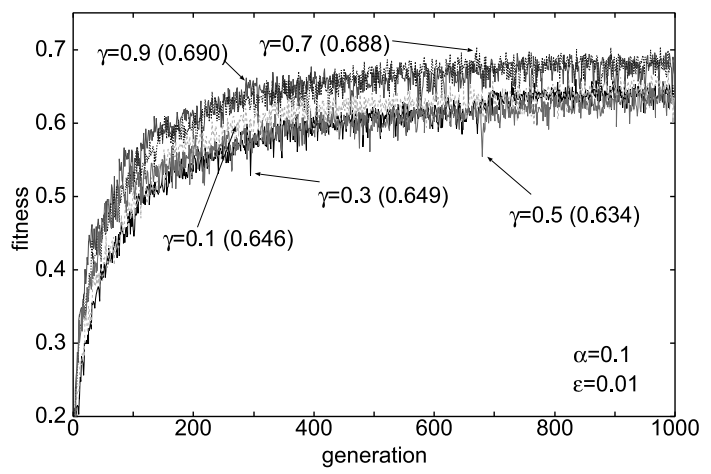

Fig. 14. effect of $\gamma$ on the fitness curves

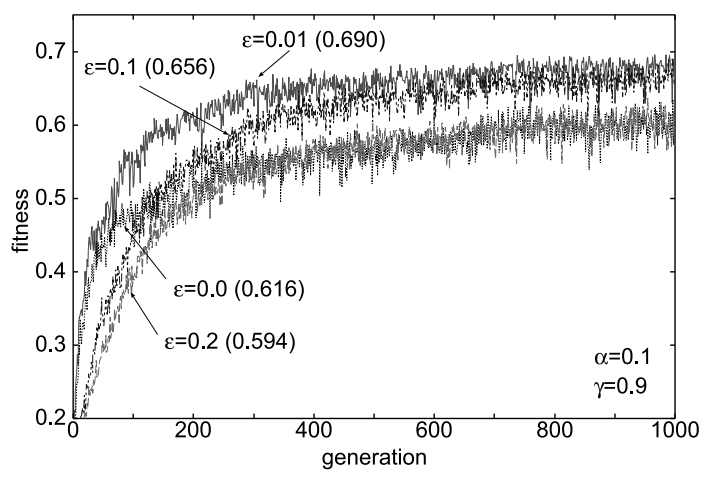

Fig. 15. effect of $\varepsilon$ on the fitness curves

step size.

Fig. 14 shows the effect of $\gamma$. If $\gamma$ is set at small value, GNP learns its $Q$ values emphasizing on near future rewards. On the other hand, if $\gamma$ is large, GNP learns them considering rewards obtained not only near but also far in the future. From Fig. 14, the larger values $(\gamma=0.7,0.9)$ show better fitness. Thus, we can find that it is necessary to update $Q$ values considering relatively far future rewards.

Fig. 15 shows the effect of $\varepsilon$. $\varepsilon=0.0$ (greedy policy) shows good result at the beginning of the generations, but the last fitness value is not good, because the random search is not carried out in RL and premature convergence occurs. The relatively large value $(\varepsilon=0.2)$ does not show good result, because the random actions are taken with high probability and cannot exploit the learned $Q$ values. In this simulation, the ini-

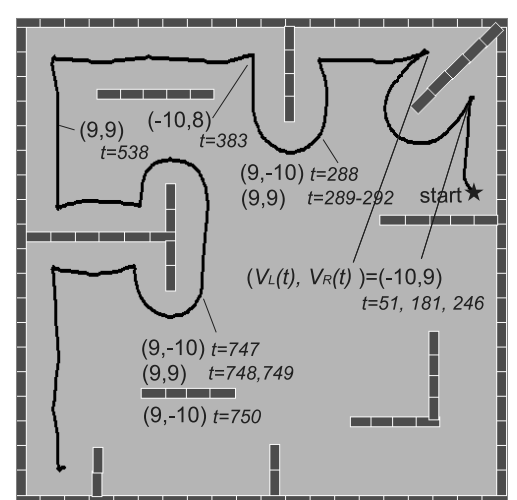

Fig. 16. Typical track of the robot controlled by GNP-RL in the inexperienced environment

Table 3. Data on the fitness obtained in an inexperienced environment

\begin{tabular}{l|ccc}
\hline & GNP-RL & S-GNP & NN-GA \\
\hline average fitness & 0.426 & 0.356 & 0.204 \\
standard deviation & 0.082 & 0.146 & 0.046 \\
\hline t-test (p-value[\%]) between & & & \\
GNP-RL and each method & - & 3.54 & $5.75 \times 10^{-10}$ \\
\hline
\end{tabular}

tial position of the robot is randomly determined, thus if the robot moves randomly with high probability, the robot cannot do appropriate judgements or processing in unknown situations and the fitness becomes low more easily than simulation I. In fact, in simulation I, the fitness of $\varepsilon=0.1$ (0.695) is better than that of $\varepsilon=0.01$ (0.647). On the other hand, $\varepsilon=0.01$ is better than 0.1 in simulation II in terms of learning speed and fitness, although the difference of the last fitness between $\varepsilon=0.01$ and 0.1 is small. Thus it can be said that 0.01 can keep the balance of exploitation and exploration in this simulation. Anyway, $\varepsilon$ has to be determined appropriately depending on the problem.

3.5.2 Generalization Ability In order to confirm the generalization ability of the proposed method, we set a robot controlled by the best program obtained in the last generation in an inexperienced environment [Fig. 16] and evaluate the fitness values.

Table 3 shows the average fitness, its standard deviation and the results of t-test (one-sided) between GNP$\mathrm{RL}$ and the other methods. The average fitness and the standard deviation in Table 3 are calculated as follows. First, calculate average fitness of 1000 trials (each trial includes 1000 steps and start position is determined randomly every trial) using the best program obtained in the first simulation in section 3.5. Next calculate average fitness using the program obtained in the second simulation. Similarly, 18 average fitness can be calculated using the best programs obtained in other 18 simulations, respectively. The average fitness and the standard deviation in Table 3 show the average and the standard deviation of the above 20 average fitness.

From Table 3, GNP-RL shows the best average fitness and there are significant differences between GNP-RL and the other methods. In addition, from Fig. 16, we can see that GNP-RL shows appropriate track. 


\section{Conclusions}

In this paper, an extended GNP-RL algorithm is proposed and applied to making khepera robot behavior. Originally, GNP can make quite compact graph structures using the ability of reusing nodes, thus the number of states could be set at small values (60 in this paper), and therefore the learning can be done efficiently. From the simulation results, the proposed method obtains higher fitness values as the generation goes on, and the robot shows the appropriate tracks for the wallfollowing problem. Especially, the proposed method shows the good fitness in the environment where the start position is changed every generation, and also shows good generalization ability in an inexperienced environment. Henceforth, we will make GNP-RL algorithm using Actor-Critic and improve its performance. Also it is necessary to apply the proposed method to various environments and compare it with other methods such as latest GPs and Neural Networks.

(Manuscript received Oct. 25, 2005, revised March 13, 2006)

\section{References}

(1) H. Katagiri, K. Hirasawa, J. Hu, and J. Murata: "Genetic Network Programming and Its Application to the Multiagent System", T. IEE Japan, Vol.122-C, No.12, pp.2149-2156 (200212) (in Japanese)

( 2 ) K. Hirasawa, M. Okubo, H. Katagiri, J. Hu, and J. Murata: "Comparison between Genetic Network Programming and Genetic Programming using Evolution of ant's Behaviors", $T$. IEE Japan, Vol.121-C, No.6, pp.1001-1009 (2001-6)

( 3 ) T. Eguchi, K. Hirasawa, J. Hu, and N. Ota: "A Study of Evolutionary Multiagent Models Based on Symbiosis", IEEE Trans. on Syst., Man and Cybernetics - Part B -, Vol.36, No.1, pp.179-193 (2006-2)

(4) R. S. Sutton and A. G. Barto: Reinforcement Learning -An Introduction-, MIT Press Cambridge, Massachusetts, London, England (1998)

(5) S. Mabu, K. Hirasawa, J. Hu, and J. Murata: "Online Learning of Genetic Network Programming", T. IEE Japan, Vol.122-C, No.3, pp.355-362 (2002-3) (in Japanese)

(6) S. Mabu, K. Hirasawa, J. Hu, and J. Murata: "Online learning of Genetic Network Programming", Proc. of 2002 Congress on Evolutionary Comput., pp.321-326, (2002-5)

( 7 ) S. Mabu, K. Hirasawa, and J. Hu: "Genetic Network Programming with Evolution and Learning and Its Application to the Tileworld Problem", T. SICE, Vol.40, No.11, pp.1105-1113 (2004-11) (in Japanese)

(8) S. Mabu, K. Hirasawa, and J. Hu: "Genetic Network Programming with Reinforcement Learning and its Performance Evaluation", 2004 Genetic and Evolutionary Computation Conference (GECCO 2004), Late Breaking Papers, (2004-6)

(9) O. Michel: Khepera Simulator Package version 2.0: Freeware mobile robot simulator written at the University of Nice Sophia-Antipolis by Olivier Michel. Downloadable from the World Wide Web at http://diwww.ep..ch/lami/team/michel/ khep-sim/ (1996)
Shingo Mabu (Member) He received the B.E. and M.E. de-

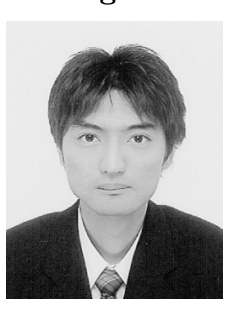
gree in Electrical Engineering from Kyushu University, Japan in 2001 and 2003, respectively, and Ph.D. degree from Waseda University, Japan. Since April 2006, he has been a visiting lecturer in Advanced Research Institute for Science and Engineering, Waseda University. Dr. Mabu is a member of the Society of Instrument and Control Engineers and IEEE.

Hiroyuki Hatakeyama (Student Member) He received

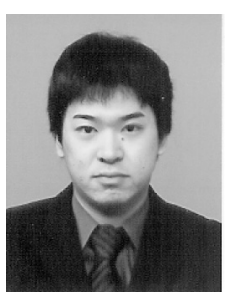
B.E. degree from Kure national college of technology in 2005. Since March 2005, he has been a master course student in Graduate School of Information, Production and Systems, Waseda University, Japan.

Moe Thu Thu (Non-member) She received the B.S. degree

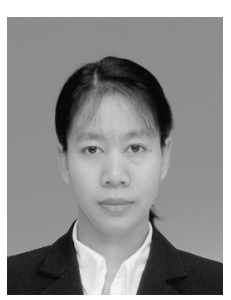
in Physics from Yangon University, Myanmar in 2000 and the M.E. degree from Waseda University, Japan in March 2006. Since April 2006, She has been a Ph.D. student in Graduate School of Information Science and Electrical Engineering, Kyushu University, Japan.

Kotaro Hirasawa (Member) He received the B.E. and M.E.

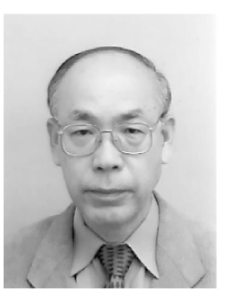
degree from Kyushu University, Japan in 1964 and 1966, respectively. From 1966 to 1992, he was with Hitachi Ltd., where he served as a vice president of Hitachi Research Laboratory. From December 1992 to August 2002, he was a Professor in Graduate School of Information Science and Electrical Engineering of Kyushu University. Since September 2002, he has been a Professor in Graduate School of Information, Production and Systems of Waseda University. Dr. Hirasawa is a member of the Society of Instrument and Control Engineers and IEEE.

Jinglu Hu (Member) He received the M.S. degree in Elec-

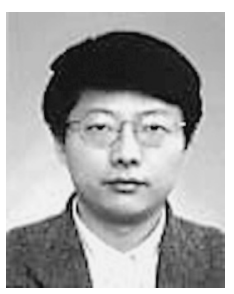
tronic Engineering from Zhongshan University, China in 1986, and the Ph.D degree in Computer Science and Engineering from Kyushu Institute of Technology, Japan in 1997. From 1986 to 1993, he worked in Zhongshan University, where he was a Research Associate and then Lecturer. From 1997 to March 2003, he was a Research Associate in Graduate School of Information Science and Electrical Engineering, Kyushu University, Japan. Since April 2003, he has been an Associate Professor in Graduate School of Information, Production and Systems of Waseda University. Dr. $\mathrm{Hu}$ is a member of the Society of Instrument and Control Engineers. 